\title{
E-MAPs: a thorough approach to networks
}

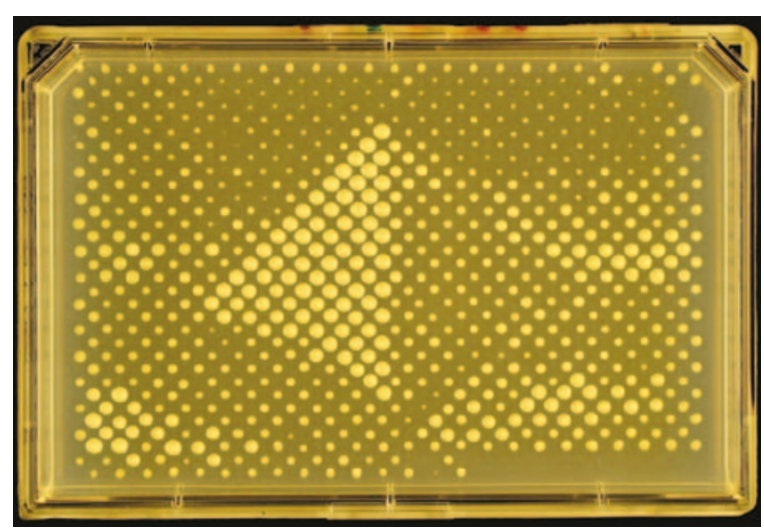

A new approach to exploring gene networks in yeast promises both a more detailed understanding of individual biological pathways and new insights into their organizational principles.

Identifying epistatic interactions is a powerful approach for defining gene functions and ordering genes

E-MAPs

provide a

powerful new

approach to

exploring

genetic interactions in yeast.... the genes that are involved in a specific process. This approach involves measuring quantitative effects on colony growth, which - unlike looking for inviability - can detect both positive and negative interactions. The high density of information that is generated allows this quantitative approach: by comparing the phenotypic effect of a particular double mutant with the effects of all other combinations seen for the two individual genes involved, an accurate measure of the direction and strength of interaction between the pair is obtained.

The authors tested their method on genes that are known to be involved in the yeast early secretory pathway. On average, each allele interacted with $3 \%$ of the others - which is a significant increase over the figure of $0.5 \%$ from wholegenome screens of non-essential genes. Schuldiner and colleagues then used hierarchical clustering to extract functional information. For each gene the E-MAP provides a profile of epistatic interactions, and genes can be clustered according to the similarity between their profiles. These clusters correspond to groups of genes that have related functions, allowing roles for poorly characterized genes to be predicted by virtue of falling into a particular cluster. For example, this indicated that three genes of previously unknown function have a role in Golgi-toendoplasmic reticulum transport - a prediction that the authors were able to validate experimentally.

The E-MAP also predicted specific functional relationships between genes. Gene pairs with the most similar phenotypic profiles never showed aggravating interactions with each other, and sometimes showed positive interactions. This is what would be expected for genes with protein products that function together in a linear pathway or complex to produce the same outcome. On the basis of this, Schuldiner and colleagues devised an algorithm to identify such gene pairs, and were again able to validate their predictions.

Finally, the E-MAP strategy provided more global information about the functional organization of the early secretory pathway. For the various secretory processes that make up the whole pathway, the authors looked at the interactions between them and compared these with the numbers that would be expected by chance. This provided a measure of the interdependence between these components, which reflected known relationships and highlighted new ones; for example, they revealed a previously unappreciated connection between lipid biosynthesis and degradation in the endoplasmic reticulum.

E-MAPs provide a powerful new approach to exploring genetic interactions in yeast in more detail than was previously possible. Applying this method to different pathways — and potentially to other organisms - promises to reveal a wealth of biological insights.

Louisa Flintoft

\section{ORIGINAL RESEARCH PAPER}

Schuldiner, M. et al. Exploration of the function and organization of the yeast early secretory pathway through an epistatic miniarray profile. Cell 123, 507-519 (2005) 